\section{Depositing thin films}

Nucleation and Growth of Thin Films. By B. Lewis and J.C. Anderson. Pp.504. (Academic: New York, San Francisco and London, 1979.) $£ 27.50 ; \$ 57$.

STUDIES of condensation of vapour atoms released in vacuum from localised sources can be dated from Knudsen's report in 1909 that mercury atoms did not condense on glass unless it was at a critical temperature, below $-140^{\circ} \mathrm{C}$; an observation reinforced by R.W. Wood who found a critical temperature effect for condensation of cadmium atoms on glass. Wood believed that the vapour atoms were reflected and Knudsen attributed this to a failure of the incident atoms to adjust to the surface temperature. Certainly the effects of reflection have been found with atomic and molecular beams and in thermal transpiration experiments, but the phenomenon witnessed by Knudsen and Wood arose from temporary sojourn of incident $\mathrm{Hg}$ and $\mathrm{Cd}$ atoms on foreign surfaces and their re-evaporation. Thus,incident atoms can thermally accommodate to a surface and still evaporate if their binding energy is weak.

It was Langmuir in 1917 who provided this latter explanation, showing by experiment and deduction that an adatom, unlike an elastically rebounding atom, would have an average surface lifetime before re-evaporating. As the lifetime rose or the impact rate increased so could the adatom density rise and migrating atoms meet to form stable clusters. Thus, onset of condensation depended on both the incident atom impact rate and the substrate temperature for a given atom/surface pair. When the surface binding forces for the adatom were high condensation ensued without these critical conditions and molecular beam experimentors some 50 years ago precoated surface detectors with silver to condense weak Cd-beams. The nucleating effect was turned to advantage by the Bosch Company in Germany who made $\mathrm{Zn}$-coated paper for capacitors using an $\mathbf{A g}$-seed layer, a process which in contemporary times has been applied to fingerprint detection.

The emergence of the electron microscope made it possible to observe the cluster growth in condensates and observe the onset of condensation with a vapour source mounted in the instrument. This technical advance and the growing interest in thin solid films in industry brought into being a world based group of 'nucleation' workers. In the same period studies of the condensation of gases, cryo-pumping, have grown and these have contributed knowledge on physical condensation mechanisms.

The book under review effectively collects together the results of studies of physical condensation during the past two decades. The authors are known for their researches on film growth and physical properties. The reference text they have produced takes the reader through the logical sequence of physical adsorption, adatom migration, cluster growth and decay, nucleation kinetics and experiments on nucleation and epitaxial growth. The book is basically concerned with the physical condensation of atoms emitted at moderate thermal energies from a separate source, that is, non-equilibrium (or vacuum) evaporation. Thus, the title could be misleading particularly as films are now deposited by many different processes involving nucleation.

Deposition by sputtering is only briefly mentioned and an example given of film growth by ion beam sputtering. The high emission energy of sputtered atoms is referred to but other sources of energy transfer, such as backscattered energetic neutrals, are not considered. (On page 3 there is some confusion in use of the phrase "fairly high vacuum pressures" to describe the operating region for sputtering. Also this is not $10^{-1}$ to 10 torr as stated but $10^{-3}$ to $10^{-1}$ torr). However, this is basically a text dealing with atom impact at low energies in vacuum and we shall probably have to wait many years before all the conditions contributing to film growth by sputtering are unravelled although much is already known.

\section{Immobilised enzymes}

Immobilized Enzymes: Research and Development. Edited by I. Chibata. Pp. 284. (Wiley: New York, London, Sydney and Toronto, 1979.) £24.50; \$46.

IN recent years there have been many books published on various aspects of immobilised eyznymes. Nevertheless, this book is a welcome addition, coming as it does from Dr Chibata and his colleagues. Dr Chibata, as Director of the Research Laboratory of Applied Biochemistry at the Tanabe Seiyaku Co. Ltd, Japan, has contributed greatly to the understanding of immobilised enzymes and cells, and to the development of industrial processes using these novel catalysts. He is therefore internationally recognised as a leading expert on the topics covered in this book.

The book contains four chapters, of which the first is a short introduction to the subject. The second chapter is devoted to the preparation of immobilised enzymes and cells, with comprehensive lists of the techniques that have been used and the enzymes and cells that have been immobilised. It concludes with a useful section on immobilised coenzymes. The next chapter describes the effects of immobilisation on the properties of enzymes and cells, such as the $p \mathrm{H}$ and temperature dependencies of their
Why do workers in this field usually refer to Frenkel for the adatom/lifetime relationship but rarely give credit for the basic concept to Langmuir and his equally important equilibrium (hyperbolic) law for adsorption? Langmuir's work is not referenced in this text.

This is not an easy work to follow - the subject is complex and full of detail - and a table defining the many symbols used would save constant back searching. Several typographical errors and some of sense occur in the text (the index in at least one case did not agree with the text and is inadequate). The book will be of value to those entering this specialist field. However, a note of warning - nucleation not only depends on substrate material and impact conditions but also on surface state. Research in this field can require ultra-high vacuum apparatus with mass spectrometers to determine the mass distribution of the incident and desorbed vapour and the residual gas composition, and instruments to determine the substrate surface condition after in situ cleaning, for example, by ion etching. It is as costly as it is complex.

L. Holland

L. Holland is a Professor in the School of Engineering and Applied Sciences, at the University of Sussex, Brighton, UK.

activities and the operational and storage stabilities in various conditions. The last and longerst chapter is concerned with the use of these catalysts in chemical and food processes, and their analytical and medical applications. Naturally, there is considerable emphasis on processes developed by the authors but it is surprising to find so little about the production of high fructose-glucose syrups, which is by far the largest industrial application of immobilised biocatalysts. The final part of this chapter covers affinity chromatography and seems rather out of place in this book.

In the preface it is stated that the aim was to produce a comprehensive text, arid to a certain extent the authors have succeeded. Unfortunately, presumably because of the interests of the authors, the book concentrates much more on the biochemical aspects and the engineering content is minimal. For instance, the section on reactors is very short and there is no consideration of the problems that arise from diffusional limiatation of the reaction rate. As a result, the book tends to be rather descriptive and lacks a quantitative approach. Despite these criticisms, this book is highly recomme: ded as a good reference text.

M. D. Lilly

M. D. Lilly is Reader in the Department of Chemical and Binchemical Engineering at University College London, UK. 